\title{
Effect of Multi-Directional Nursing Intervention on the Prognosis and Quality Of Life in Patients with Type 2 Diabetes
}

\author{
YE. FU, MEIHUA TAN¹ AND MULI FU* \\ Department of Gastroenterology, Department of Infectious Diseases, The First Affiliated Hospital, University of South China, \\ No. 69 Chuanshan Road, Hengyang, Hunan 421001, China, ${ }^{1}$ Department of Endocrine, Affiliated Nanhua Hospital, University \\ of South China, No. 336, Dongfeng South Road, Hengyang, Hunan 421002, China
}

Fu et al.: Effect of Multi-Directional Nursing Intervention in Type 2 Diabetes Patients

\begin{abstract}
To explore the effect of the multi-directional nursing intervention on the complications and nursing satisfaction of patients with Type 2 diabetes. Eighty-eight patients with type 2 diabetes treated in the endocrinology department of our hospital from June 2018 to June 2019 were collected and randomly divided into the control group $(n=44)$ and experimental group $(n=44)$. Patients with type 2 diabetes in the control group received routine medical care. The experimental group received a multi-directional nursing intervention. The nursing effect and nursing satisfaction of the two groups were evaluated after $12 \mathrm{w}$. The results showed that after $12 \mathrm{w}$ of intervention, the multi-directional nursing intervention had a positive effect on the related indexes or risk factors of type 2 diabetes complications, including Hemoglobin A1C, blood pressure, and Low-density lipoprotein cholesterol level, and the improvement effect was significantly better than that in the control group. After the intervention, the scores of self-management ability in the two groups were higher than those before the intervention, and the scores in the experimental group were higher than those in the control group. And the treatment compliance of patients in the experimental group was significantly higher than that in the control group, the incidence of complications in the experimental group was significantly lower than that in the control group $(\mathbf{p}<\mathbf{0 . 0 5})$. In addition, the average nursing satisfaction of the experimental group was higher than that of the control group, and the difference between the two groups was statistically significant $(p<0.05)$. The multi-directional nursing intervention model is effective in patients with type 2 diabetes and should be widely used in the clinic to help more patients with diabetes self-management education.
\end{abstract}

Key words: Type 2 diabetes; Nursing intervention; Low-density lipoprotein cholesterol (LDL-C); the multi-directional nursing intervention

Diabetes was a global health problem. It was a metabolic disease characterized by chronic hyperglycemia accompanied by the disorders of carbohydrate, fat, and protein metabolism. Diabetes determines a person's risk of diabetic complications or secondary diseases $^{[1,2]}$, which were divided into two categories, macro-vascular complications (coronary heart disease, cerebrovascular diseases) and micro-vascular complications (retinopathy, peripheral neuropathy, and nephropathy $)^{[3]}$. Unfortunately, there was a high prevalence of diabetic complications in patients with type 2 diabetes in some countries. The etiology of common complications or secondary diseases in patients with type 2 diabetes was complex, and the incidence of the diabetic complications in patients with longer medical history was higher than that in patients with shorter medical history ${ }^{[4]}$. Without appropriate intervention, these destructive complications may lead to the cognitive impairment, unemployment, poor quality of life ${ }^{[5]}$, and poor nursing of patients ${ }^{[6]}$.

Studies have shown that the diabetic complications, especially macro-vascular complications, were associated with the ignorance of patients ${ }^{[7]}$, failure to comply with the diabetes management rules ${ }^{[8]}$, and poor blood glucose control, long medical history ${ }^{[9]}$, and the lack of medical care ${ }^{[10]}$. The incidence of macrovascular complications can be reduced by strict control of blood glucose, blood pressure, and low density 
lipoprotein-cholesterol (LDL-C) levels ${ }^{[11]}$. However, some studies have shown that many patients with type 2 diabetes cannot achieve the desired therapeutic effect because of the above reasons ${ }^{[12,13]}$. Although most of the nursing services for the type 2 diabetes patients in China were from their own community hospitals ${ }^{[14]}$, it cannot prevent the diabetic complications due to it may encounter many obstacles in providing structured care. The detection and control process of blood glucose was mainly based on the fasting plasma glucose value (FPG). On the other hand, few units evaluate Hemoglobin A1C (HbA1c), because most of the primary care institutions cannot detect this indicator ${ }^{[14]}$.

Nurses were at the forefront of the health care services and played an important role in improving the quality of nursing ${ }^{[15]}$. In the health care system, the nurses were usually required to diagnose and treat the common health problems $(88.2 \%)$, as well as to provide health services for the elderly $(87.9 \%)$ and continuous care for patients with chronic diseases $(85.4 \%)^{[16]}$. Depending on the nature of the task, to achieve the therapeutic effectiveness, the primary health care services can provide the routine interventions such as blood glucose self-management education for the type 2 diabetes patients. This study aims to evaluate the effect of the multi-directional nursing intervention on the complications of type 2 diabetes and the nursing satisfaction of patients with type 2 diabetes. The specific plan was as follows: The type 2 diabetic patients were divided into two groups, receiving the multi-directional nursing intervention and routine nursing intervention respectively, and evaluated by the relevant indicators of diabetic complications, including HbA1c, blood pressure, and LDL-C level; Compared with the satisfaction of type 2 diabetic patients who received the multi-directional nursing intervention and routine nursing intervention.

\section{MATERIALS AND METHODS}

\section{Clinical data:}

A total of 90 patients with type 2 diabetes treated in the endocrinology department of our hospital from June 2018 to June 2019 were collected. Inclusion criteria: The patient meets the diagnostic criteria of WHO diabetes in 1999 and the diagnostic criteria of type 2 diabetes in 2013 China Diabetes Guide; The patient has complete clinical diagnosis and treatment data; The course of type 2 diabetes was more than 6 mo; The patient has clear consciousness, normal language expression and eating, no serious complications, no hypoglycemia inducing factors such as low food intake, excessive exercise, and drug overdose. Exclusion criteria: Patients with severe hepatic and renal metabolic dysfunction; Patients with metastatic malignant tumors; Patients with a history of mental illness or family history. Finally, 88 patients were included in this study. According to the principle of random control, the patients were randomly divided into the control group $(\mathrm{n}=44)$ and treatment group $(n=44)$.

Patients with type 2 diabetes in the control group $(n=44)$ received routine care, while those in the experimental group $(\mathrm{n}=44)$ received the multi-directional nursing intervention. The multi-directional nursing intervention was a self-management education through training, which aims to explain the necessity of diabetes selfmanagement to patients with type 2 diabetes. The goals of the intervention were as follows, to provide education and educational support to the patients (cognitive part); to help the patients integrate diet and exercise into daily management advice (behavioral component); to provide psychological support for patients to change their roles, interpersonal relationships and emotions (emotional components).

In the experimental group, each nurse had the one-onone meeting with the patients and instructed the patients to change their behavior by telephone (TABLE 1). Each instruction was tailored to the needs of each patient and collects information for the next stage of training. The intervention will explain the self-management of diabetes to the patients, including the diet control, exercise, oral hypoglycemic drugs and side effects, stress, self-monitoring, hygiene and foot care. Some participants also received diabetes self-management manuals. During each instruction, the nurse also measured the blood pressure and postprandial blood glucose levels, and assessed the self-management and behavioral changes of the diabetic patients. The nurses also provide the emotional support and use motivational interviews or open Q \& A to help the patients solve personal problems (3 times every two w until w 8) and telephone guidance every two w (until w12).

\section{Evaluation criteria:}

The researchers assessed the patients' diabetes knowledge and self-management using the diabetes' knowledge questionnaire and diabetes self-care activity questionnaire ${ }^{[17]}$. Blood samples were collected before the beginning of the multi-directional nursing intervention (before the trial) and after the final guidance intervention at the end of the 12th w (after the 


\begin{tabular}{ll}
\hline Item & Content \\
\hline Evaluate & $\begin{array}{l}\text { Find out the trajectory of individual diabetes diagnosis, treatment and impact on life. An individual's } \\
\text { drugs used, stress and foot care, values, establishment and customization of personal diabetes self- } \\
\text { management education (cognitive education) }\end{array}$ \\
Definition & $\begin{array}{l}\text { The definition of the behavioral goals, focusing on the behavioral changes in diet, exercise, drugs, stress, } \\
\text { self-monitoring and foot care. }\end{array}$ \\
Explore & What is the current situation of individuals in defining goals? \\
Action projects & Define options, what are the different options designed to achieve the goal? \\
Study & The patient cooperates and promises to take action. \\
Feedback & Implement the agreed action plan for 2 w \\
& During follow-up or on-site visits, the nurses discuss with patients what they have learned? How does \\
\end{tabular}

trial) to detect the concentration of HbA1c and LDL-C. The researchers collected the data at the beginning of the study and at the end of $12 \mathrm{w}$.

The satisfaction questionnaire of the nursing intervention was a method to evaluate patients' perception of nursing. The scale was mainly scored according to subjective impression ${ }^{[18]}$. There were four dimensions of satisfaction, including feasibility, accessibility, hospitalization, and acceptability and a total of 21 items. The reliability of the questionnaire was 0.92 .

Treatment compliance. Treatment compliance was carried out in the form of investigation and evaluation, which was scored from 3 aspects, compliance, partial compliance, and non-compliance. The total score was 100 , the higher the score, the better the compliance. The incidence of complications. The complications were angina pectoris and hypoglycemia.

After $12 \mathrm{w}$ of intervention, the related indexes or risk factors of type 2 diabetes complications were evaluated in the multi-directional nursing intervention group.

The dietary compliance of patients after the intervention was evaluated by the dietary behavior compliance scale with a total score of $100 \leq 60$ means non-compliance, 60-95 means partial compliance, $>95$ means compliance, the total compliance rate $=($ cases of partial compliance + cases of compliance)/total cases $\times 100 \%$.

The self-management ability score was designed according to the standard of the chronic disease selfmanagement research scale, including blood glucose monitoring, exercise management, medication management and diet management. Three dimensions of blood glucose monitoring, exercise and medication management were selected, the higher the score, the better the ability of self-management.

\section{Statistical analysis:}

SPSS 20.0 software was sued for statistical analysis (frequency and percentage data). Independent or nonindependent sample t-test was used to compare the HbA1c level, average blood pressure, LDL-C level, and satisfaction score between the two groups. $p<0.05$ means the difference was significant.

\section{RESULTS AND DISCUSSION}

The results showed that after $12 \mathrm{w}$ of intervention, the multi-directional nursing intervention had a positive effect on the related indexes or risk factors of type 2 diabetes complications, including $\mathrm{HbA1c}$, blood pressure, and LDL-C level (TABLE 2), and the improvement effect was significantly better than that of the control group $(\mathrm{p}<0.05)$.

The results showed that different degrees of complications occurred in both the control group and the experimental group. In the control group, 8 patients had complications, including 3 cases of angina pectoris and 5 cases of hypoglycemia. The incidence of complications was $18.18 \%$. Complications occurred in 2 patients in the experimental group, including 0 cases of angina pectoris and 2 cases of hypoglycemia, and the incidence of complications was $4.55 \%$. The incidence of complications in the experimental group was lower than that in the control group, and there was a significant difference between the two groups (fig. $1, \mathrm{p}<0.05$ ).

The average satisfaction of nursing intervention was shown in fig. 2. The average nursing satisfaction $(\mathrm{Xexp}=4.91, \mathrm{SD}=0.18)$ in the experimental group was higher than that of the control group (Xcont $=2.49$, $\mathrm{SD}=0.91$ ), and there was a significant difference between the two groups $(\mathrm{p}<0.05)$. 
In the control group, there were 10 cases of noncompliance, 9 cases of partial compliance, and 25 cases of complete compliance, with a total compliance rate of $77.27 \%$ (34/44). In the experimental group, there were 2 cases of non-compliance, 13 cases of partial compliance, and 29 cases of complete compliance, with a total compliance rate of $95.46 \%(42 / 44)$. The total

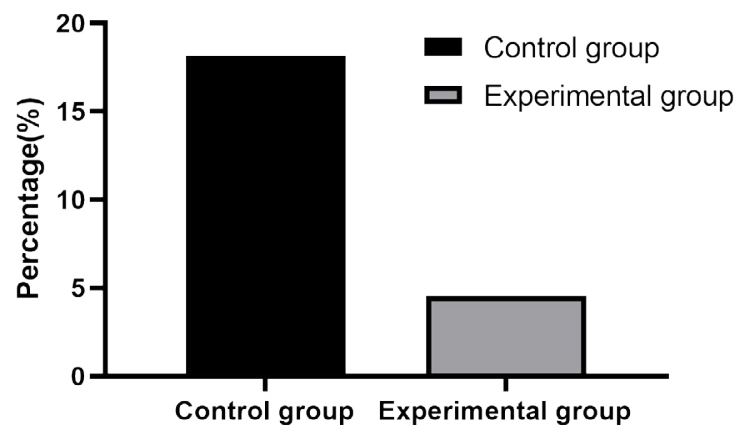

Fig. 1: Incidence of complications in two groups compliance rate of the experimental group was higher than that of the control group (fig. 3), and the difference was statistically significant $\left(\chi^{2}=6.531, \mathrm{p}=0.011\right)$.

There was no significant difference in the scores of self-management ability between the two groups before the intervention $(p>0.05)$. After the intervention, the scores of self-management ability in the two groups were higher than those before the intervention, and the scores in the experimental group were higher than those in the control group, and the difference was statistically significant (TABLE $3, \mathrm{p}<0.05$ ).

This study evaluates the effect of the multi-directional nursing intervention, which addresses issues related to the self-management of diabetes. A large number of studies have shown that the patients with type 2 diabetes often lack the awareness of the diabetes selfmanagement, and lack the awareness of the prevention of macrovascular and microvascular complications

TABLE 2: RELATED BLOOD INDEX PARAMETERS OF DIABETES IN TWO GROUPS BEFORE AND AFTER TREATMENT

\begin{tabular}{|c|c|c|c|c|c|c|}
\hline \multirow{2}{*}{ Dependent variables } & \multicolumn{3}{|c|}{ Control group $(n=44)$} & \multicolumn{2}{|c|}{ Experimental group $(n=44)$} & \multirow{2}{*}{$\mathbf{P}$} \\
\hline & & & SD & $x$ & SD & \\
\hline \multirow[t]{2}{*}{ HbA1c level } & Before & 8.17 & 0.99 & 8.21 & 1.41 & 0.877 \\
\hline & After & 7.72 & 0.97 & 7.10 & 0.67 & $0.037^{a}$ \\
\hline \multicolumn{7}{|l|}{ Blood pressure level } \\
\hline \multirow[t]{2}{*}{ Systolic pressure level } & Before & 133.00 & 14.90 & 132.00 & 10.56 & 0.833 \\
\hline & After & 127.40 & 15.30 & 121.00 & 10.28 & $0.032^{\mathrm{a}}$ \\
\hline \multirow[t]{2}{*}{ Diastolic pressure level } & Before & 85.00 & 9.46 & 84.50 & 8.87 & 0.905 \\
\hline & After & 79.40 & 19.44 & 81.30 & 9.10 & $0.045^{\mathrm{a}}$ \\
\hline \multirow[t]{2}{*}{ LDL-C level } & Before & 139.30 & 29.61 & 138.75 & 40.39 & 0.855 \\
\hline & After & 110.4 & 25.60 & 123.60 & 45.54 & $0.021^{a}$ \\
\hline
\end{tabular}

a means the difference in the two groups before and after treatment was statistically significant.
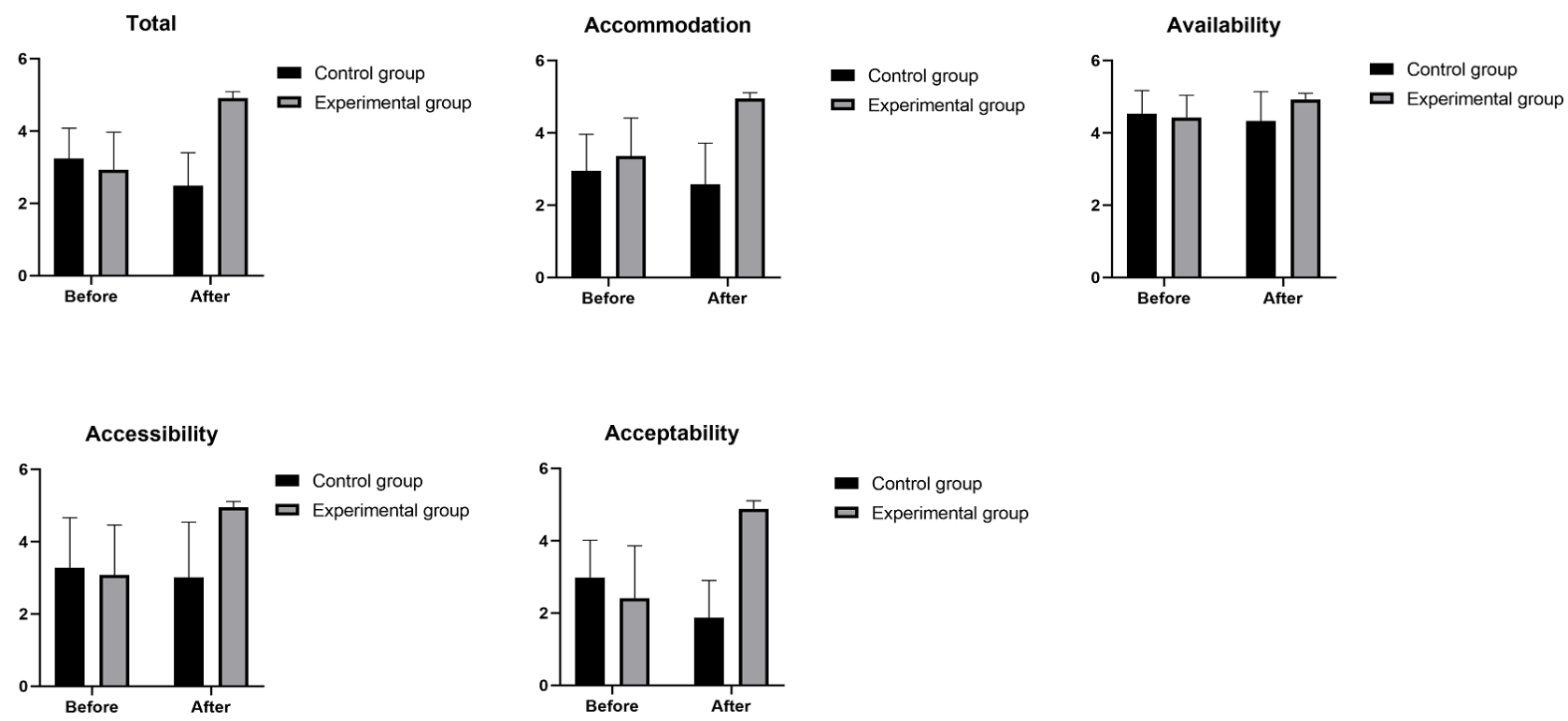

Fig. 2: Evaluation of nursing satisfaction in two study groups 
TABLE 3: SELF-MANAGEMENT ABILITY OF PATIENTS IN TWO GROUPS

\begin{tabular}{|c|c|c|c|c|c|c|c|}
\hline \multirow{2}{*}{ Groups } & \multirow{2}{*}{ Cases } & \multicolumn{2}{|c|}{ Blood glucose monitoring } & \multicolumn{2}{|c|}{ Medication management } & \multicolumn{2}{|c|}{ Sports management } \\
\hline & & Before & After & Before & After & Before & After \\
\hline Control group & 44 & $30.40 \pm 5.70$ & $35.24 \pm 4.21$ & $11.25 \pm 2.35$ & $12.80 \pm 2.01$ & $15.30 \pm 4.10$ & $21.20 \pm 5.05$ \\
\hline Experimental group & 44 & $30.50 \pm 5.10$ & $48.90 \pm 3.30$ & $11.40 \pm 2.60$ & $18.90 \pm 1.50$ & $15.50 \pm 3.90$ & $38.80 \pm 4.20$ \\
\hline
\end{tabular}

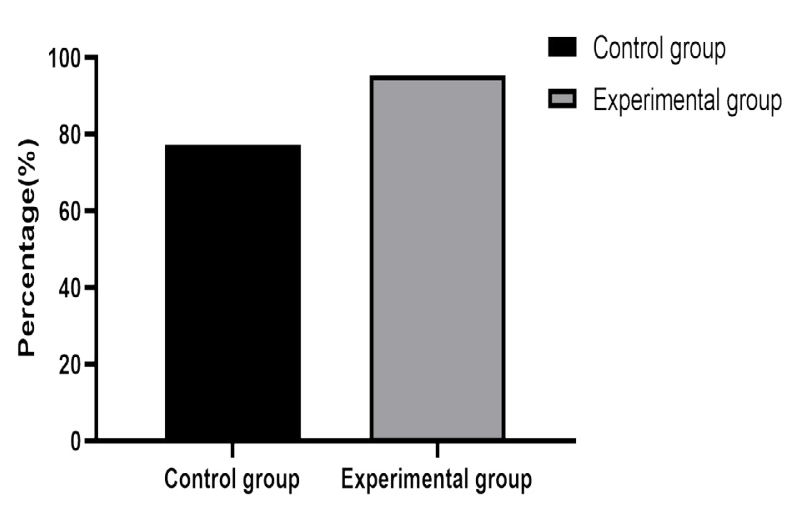

Fig. 3: Compliance comparison between two groups

in daily life ${ }^{[19-21]}$. In addition, patients with type 2 diabetes were at higher risk of related complications, such as cardiovascular disease, retinopathy, and neuropathy ${ }^{[19,22]}$. Diabetic nephropathy can result from increased glomerular capillary flow that, in turn, results in increased extracellular matrix production and endothelial damage. Inadequate blood glucose control lead to structural nerve damage including segmental demyelination, axonal atrophy and progressive demyelination. This can lead to neuropathy. In general, the multi-directional nursing intervention focuses on these major issues, and providing feasible strategies for strengthening knowledge and increasing management advice.

This finding suggests that training can change the behavior of the patients with chronic diseases, such as asthma and the elderly ${ }^{[23,24]}$. The reason for choosing intervention and diabetes self-management education was that when patients encounter difficulties in maintaining a complex life pattern for a long time, they can increase the power of behavior change and provide continuous support in the process of training. This study found that for patients with type 2 diabetes, the reduction of $\mathrm{HbAlc}$ has greater benefits in reducing diabetic complications as it unveils the blood glucose control of the preceding three months. The average decrease of $\mathrm{HbA1c}$ in the observation group was 96 $\%$, while that in the control group was only $45 \%$. At the end of the intervention, the HbAlc levels of all patients in the intervention group were lower. If continuous intervention, the extent of $\mathrm{HbA} 1 \mathrm{c}$ reduction may significantly affect the mortality and morbidity of patients with type 2 diabetes. Studies have found that
HbAlc was reduced by $1 \%$, diabetic micro-vascular complications (such as retinopathy and nephropathy) can be reduced by 30-35\%, and the mortality can be reduced by $28 \%$. Although $\mathrm{HbAlc}$ was reduced by $0.965 \%$ in this study, which is almost the same level as recommended by Diabetes control and complications trial (DCCT) ${ }^{[20]}$. Finally, in view of the necessity of replication in this study, future studies should explore whether its activity persists at the end of the multidirectional nursing intervention.

The multi-directional nursing intervention has an effect on blood pressure. After the intervention, the average blood pressure level of patients receiving the multidirectional nursing intervention was significantly lower than that of patients receiving routine care. The reason may be related to the hypertension in the patients ${ }^{[21]}$. Atherosclerosis leads to the vascular wall hardening and hemodynamic problems. Hypertension was the most common complication in diabetes ${ }^{[25]}$, which was closely related to cardiovascular and micro-vascular ${ }^{[26]}$. Moreover, most of the patients with type 2 diabetes in this study have mild hypertension, a wide range of age and long-term diabetes. Therefore, our results show that the multi-directional nursing intervention is suitable for patients with hypertension complicated with type 2 diabetes. The multi-directional nursing intervention measures have an obvious effect on LDL-C. Through the training of nurses, patients can choose their own training topics, and they often choose topics related to the level of LDL-C, which was related to atherosclerosis ${ }^{[24]}$. Therefore, the change of LDL-C level can reflect the improvement of patients with type 2 diabetes. At the same time, we found that after the multi-directional nursing intervention, the incidence of complications in patients was significantly lower than that in the control group. In addition, the multidirectional nursing intervention can also improve the treatment compliance of patients, which is conducive to the recovery of patients.

After the intervention, the average score of nursing service satisfaction in the experimental group was significantly higher than that in the control group. After the evaluation of each item of the questionnaire, the average score was higher after the intervention. This means that the patients with type 2 diabetes were 
satisfied with the nursing intervention. This result is similar to Duangla's study ${ }^{[27]}$, nursing services with volunteer participation can make it easier for the type 2 diabetes patients enter the diabetes clinics, and improve the patients' nursing satisfaction and self-management ability. It was clear that the multi-directional nursing intervention provides patients with easier access to services and receives guidance on the process and diabetes self-management information. All of these were valued by most participants and considered to be very useful.

This multi-directional nursing intervention model is effective in patients with type 2 diabetes and should be widely used in the clinic to help more patients with diabetes self-management education.

\section{Authors' contributions:}

Ye Fu conceived and designed the experiments; Meihua Tan performed the experiments; Muli Fu analyzed the data and wrote the paper.

\section{Acknowledgements:}

This work was supported by The First Affiliated Hospital, University of South China.

\section{Conflicts of interest:}

The authors report no conflicts of interest.

\section{REFERENCES}

1. World Health Organization. The world health report 2006-working together for health. WHO 2006.

2. World Health Organization. Definition, diagnosis and classification of diabetes mellitus and its complications: Report of a WHO consultation. Part 1, Diagnosis and classification of diabetes mellitus. WHO 1999.

3. Stratton IM, Adler AI, Neil HA, Matthews DR, Manley SE, Cull CA, et al. Association of glycaemia with macro-vascular and micro-vascular complications of Type 2 diabetes (UKPDS 35): prospective observational study. BMJ 2000;321:405-12.

4. Chetthakul T, Deerochanawong C, Suwanwalaikorn S, Kosachunhanun N, Ngarmukos C, Rawdaree P, et al. Thailand diabetes registry project: prevalence of diabetic retinopathy and associated factors in type 2 diabetes mellitus. J Med Assoc Thai 2006;89:S27-36.

5. Leelawattana R, Pratipanawatr T, Bunnag P, Kosachunhanun $\mathrm{N}$, Suwanwalaikorn S, Krittiyawong S, et al. Thailand diabetes registry project: prevalence of vascular complications in longstanding type 2 diabetes. J Med Assoc Thai 2006;89:S54-9.

6. Lloyd A, Sawyer W, Hopkinson P. Impact of long-term complications on quality of life in patients with type 2 diabetes not using insulin. Value Health 2001;4:392-400.

7. De Berardis G, Pellegrini F, Franciosi M, Belfiglio M, Di Nardo B, Greenfield S, et al. Quality of diabetes care predicts the development of cardiovascular events: results of the QuED study. Nutr Metab Cardiovasc Dis 2008;18:57-65.
8. Gingkokgraud T. Relationships between personal factors, knowledge related to diabetes, family and community supports with self-care performance of diabetic patients, community hospitals, Kalasin Province. Chulalongkorn University; 1997.

9. Rittichu C. Patient adherence to regimens and glycemic control in type 2 diabetes. Mahidol University; 2002.

10. McAlpine RR, Morris AD, Emslie-Smith A, James P, Evans JM. The annual incidence of diabetic complications in a population of patients with Type 1 and Type 2 diabetes. Diabet Med 2005;22:348-52.

11. Schellhase KG, Koepsell TD, Weiss NS. Glycemic control and the risk of multiple microvascular diabetic complications. Fam Med 2005;37:125-30.

12. Hansson L, Zanchetti A, Carruthers SG, Dahlof B, Elmfeldt $\mathrm{D}$, Julius S, et al. HOT Study Group. Effects of intensive blood-pressure lowering and low-dose aspirin in patients with hypertension: principal results of the Hypertension Optimal Treatment (HOT) randomised trial. Lancet 1998;351:1755-62.

13. Harris MI, Eastman RC, Cowie CC, Flegal KM, Eberhardt MS. Racial and ethnic differences in glycemic control of adults with type 2 diabetes. Diabetes Care 1999;22:403-8.

14. Beaton SJ, Nag SS, Gunter MJ, Gleeson JM, Sajjan SS, Alexander CM. Adequacy of glycemic, lipid, and blood pressure management for patients with diabetes in a managed care setting. Diabetes Care 2004;27: 694-8.

15. Nitiyanant W, Chetthakul T, Sang Ak, Therakiatkumjorn C, Kunsuikmengrai K, Yeo JP. A survey study on diabetes management and complication status in primary care setting in Thailand. J Med Assoc Thai 2007;90:65-71.

16. Hanuchururnkul S. Nursing in health care reform. Int J Nurs Pract 2001;7:365.

17. Hanucharurnkul S. Nurses in primary care and the nurse practitioner role in Thailand. Contemp Nurse 2007;26:83-93.

18. Aekplakorn W, Suriyawongpaisal P, Sirirassamee B. Assessment of capacity for cardiovascular disease control and prevention in Thailand: a qualitative study. Southeast Asian J Trop Med Public Health 2005;36:741-7.

19. Funnell MM, Haas LB. National Standards for Diabetes Self-Management Education Programs. Diabetes Care 1995;18:100-16.

20. Ratanasuwan T, Indharapakdi S, Promrerk R, Komolviphat T, Thanamai Y. Health belief model about diabetes mellitus in Thailand: the culture consensus analysis. J Med Assoc Thai 2005;88:623-31.

21. Rachmani R, Levi Z, Slavachevski I, Avin M, Ravid M. Teaching patients to monitor their risk factors retards the progression of vascular complications in high-risk patients with Type 2 diabetes mellitus? A randomized prospective study. Diabet Med 2002;19:385-92.

22. Rawdaree $\mathrm{P}$, Ngarmukos $\mathrm{C}$, Deerochanawong C, Suwanwalaikorn S, Chetthakul $\mathrm{T}$, Krittiyawong $\mathrm{S}$, et al. Thailand diabetes registry (TDR) project: clinical status and long term vascular complications in diabetic patients. J Med Assoc Thai 2006;89:S1-9.

23. Rerkasem K, Kosachunhanum N, Chongruksut W, Koiam W, Limpijarnkit L, Chimplee K, et al. A hospital-based survey of risk factors for diabetic foot ulceration in northern Thailand. Int J Low Extrem Wounds 2004;3:220-2.

24. Bennett JA, Perrin NA, Hanson G, Bennett D, Gaynor W, Flaherty-Robb M, et al. Healthy aging demonstration project: nurse coaching for behavior change in older adults. Res Nurs Health 2005;28:187-97.

25. Smith SR, Jaffe DM, Highstein G, Fisher EB, Trinkaus KM, 
Strunk RC. Asthma coaching in the pediatric emergency department. Acad Emerg Med 2006;13:835-9.

26. Denver EA, Barnard M, Woolfson RG, Earle KA. Management of uncontrolled hypertension in a nurse-led clinic compared with conventional care for patients with type 2 diabetes. Diabetes Care 2003;26:2256-60.

27. Duangla N. Effect of nursing service with volunteers' participation on satisfaction and self-care of diabetic patients in a diabetic clinic. Chulalongkorn University; 2002.
This is an open access article distributed under the terms of the Creative Commons Attribution-NonCommercial-ShareAlike 3.0 License, which allows others to remix, tweak, and build upon the work non-commercially, as long as the author is credited and the new creations are licensed under the identical terms

This article was originally published in a special issue,

"Biomedical Research in Clinical and Preclinical

Pharmaceutics" Indian J Pharm Sci 2020:82(5) 